\section{Weniger Ansteckungen durch verbesserte Handhygiene in Pflegeheimen}

\begin{abstract}
Das Norovirus, eine der wichtigsten Ursachen für Gastroenteritis, verursacht bei Bewohnern von Pflegeheimen eine höhere Morbidität und Mortalität als in der Allgemeinbevölkerung. Eine Arbeitsgruppe aus Frankreich analysierte mit Hilfe eines modernen, Daten-getriebenen, mathematischen Modells die Wirksamkeit von einfachen Interventionen zur Kontrolle von Norovirus-Ausbrüchen in Pflegeheimen.
\end{abstract}

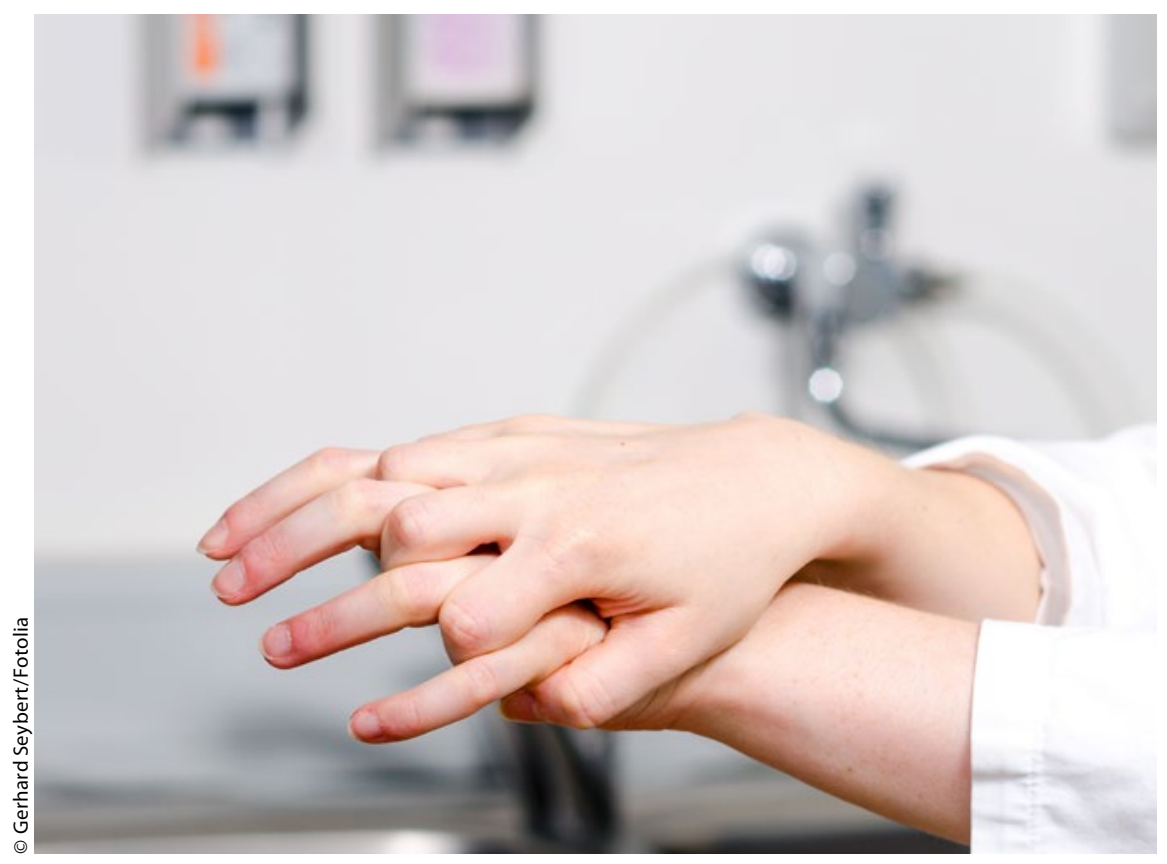

$\mathrm{N}$ ach dem Modell, bei dem zunächst die in Studien gezeigte Handhygiene-Compliance-Rate des Pflegepersonals von $15 \%$ zugrunde gelegt wurde, treten bei einem Norovirus-Ausbruch in französischen Pflegeheimen innerhalb von 100 Tagen im Durchschnitt 19 Gastroenteritis-Fälle auf. Dies stimmt mit publizierten Inzidenzraten in Pflegeheimen überein. Das Isolieren von symptomatischen Patienten erwies sich als sehr effektiv: Die Maßnahme reduzierte die Zahl der simulierten Krankheitsfälle um $88 \%$. Auch eine verbesserte Handhygiene des Personals wirkte sich erheblich aus: Setzten $60 \%$ der Pflegekräfte die empfohlenen Hygienevorschriften um, nahm die simulierte Zahl der Erkrankten um $75 \%$ ab. Eine verbesserte Handhygiene der Bewohner korrelierte zwar linear mit einer Abnahme der Erkran- kungsfälle, doch die erzielten Effekte waren weniger ausgeprägt.

Für die Studie wurden Daten von Bewohnern und Pflegepersonal eines französischen Pflegeheims mit 100 Betten erfasst und hierauf basierend ein spezielles mathematisches Modell zur Simulation der Entwicklung eines Norovirus-Ausbruchs entwickelt. Mit Hilfe des Modells simulierten die Autoren den Einfluss von drei Interventionen innerhalb von 100 Tagen. Bei den Interventionen handelte es sich um eine verbesserte Handhygiene des Pflegepersonals bzw. der Bewohner sowie um die Isolierung von symptomatischen Bewohnern.

Nach den Ergebnissen dieser Analyse können einfache Maßnahmen dazu beitragen, einen Norovirus-Ausbruch in Pflegeheimen zu kontrollieren. Sie unterstreichen eindrücklich die Bedeutung der Handhygiene des Pflegepersonals. Könnte die Compliance von den publizierten $15 \%$ auf $60 \%$ erhöht werden, würde dies zu einer effektiven Kontrolle von Norovirus-Ausbrüchen in Pflegeheimen beitragen.

(pe)

Assab $R$, et al. The role of hand hygiene in controlling norovirus spread in nursing homes. BMC Infectious Diseases (2016) 16:395

\section{Kommentar}

Im realen Pflegealltag können Verbesserungen in der Händehygiene nicht simuliert, werden. Sie müssen durch Interventionen erreicht werden. Die Compliance-Rate steigt dabei umso höher je niedriger die Ausgangsrate ist. Die französische Studie geht in ihren Berechnungen von einer erschreckend niedrigen Compliance des Personals bei der Händehygiene von nur 15\% aus. Die Zahlen stammen aus einer amerikanischen Studie von 2008, die fast 500 Händehygienegelegenheiten in zwei Pflegeheimen beobachtete und zudem auch noch eine durchschnittliche Einwirkzeit von nur 15 Sekunden belegte.

Zu einem signifikanten Rückgang von Infektionen kommt es anderen Studien zufolge erst ab einer Compliance-Rate von durchschnittlich $80 \%$. Auch ist nachgewiesen, dass eine Händedesinfektion unter $30 \mathrm{Se}$ kunden keinen ausreichenden Schutz bietet. WHO und KRINKO empfehlen nachdrücklich multimodale Programme zur Händehygiene. Im Fokus: Händedesinfektionsmittel am Point of Care, Schulung und Training, Monitoring und Feedback sowie verbesserte Workflows. Compliance-Raten von $15 \%$, wie in der Studie angegeben, sind fast schon als grobe Fahrlässigkeit zu betrachten. Deshalb sollte jede Einrichtung erstens ihre eigene Compliance-Rate ermitteln und zweitens die Händehygiene zur Chefsache machen. Nur so können die Versorgungsqualität und Bewohnersicherheit dauerhaft verbessert werden.

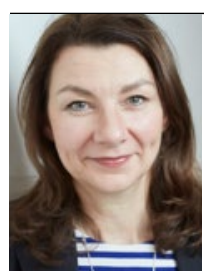

Sabine Niknam Schmilinskystraße 32 20099 Hamburg 